\title{
6 Fragestellung/Hypothesen
}

Da es bisher noch keine Untersuchung zur „Emotionale Intelligenz“ in ihrer heutigen Bedeutung bei bipolar affektiven Störungen gibt, wurde die vorliegende empirische Studie darauf ausgerichtet, einerseits zu ermitteln, ob und auf welcher Weise „Emotionale Intelligenz“ bei bipolaren Patienten mit Erkrankungsmerkmalen zusammenhängt; andererseits wurde die Ausprägung von „Emotionale Intelligenz“ bei manisch depressiven Patienten und gesunden Kontrollpersonen verglichen. Da im deutschen Sprachraum noch keine vergleichbare Studie zur „Emotionale Intelligenz“ bei bipolaren Störungen vorliegt, wurde hier erstmalig eine standardisierte Befragung zur Erhebung eingesetzt und mit gesunden Kontrollpersonen verglichen.

Aus der Forschungsliteratur und aus der klinischen Praxis wissen wir, dass die sozialen Funktionen bipolarer Patienten beeinträchtigt sind. Deshalb wird die Annahme gestellt, dass einerseits das allgemeine soziale Funktionsniveau und andererseits die „Emotionale Intelligenz“ bei erkrankten Patienten im Vergleich zur Kontrollgruppe verringert sind. Auch wird angenommen, dass diese beiden Variablen zueinander in positivem Zusammenhang stehen.

Im Rahmen dieser Studie soll auch untersucht werden, ob der momentane affektive Zustand der aktuell stabilen Patientengruppe, nämlich a), die „Emotionale Intelligenz“ und b), das soziale Funktionsniveau beeinflusst. Es wird angenommen, dass das Funktionsniveau und die gesamte „Emotionale Intelligenz“ der Patienten umso niedriger sind, je mehr die aktuelle Stimmung der Patientengruppe depressive Tendenzen zeigt.

Da es die „Emotionale Intelligenz“ durch Training verbessert werden kann, wurde angenommen dass diese mit dem Lebensalter und ev. mit der Erkrankungsdauer in positivem Zusammenhang steht. Je höher das Lebensalter (a), bzw. je länger die Erkrankungsdauer ist (b), umso höher sollte der Gesamtwert der „Emotionale Intelligenz" sein. 